OPEN ACCESS

Edited by:

Kyung-Woo Lee,

Konkuk University, South Korea

Reviewed by:

Guang-Hai Qi,

Feed Research Institute (CAAS), China Samiru Sudharaka Wickramasuriya,

Chungnam National University,

South Korea

*Correspondence: Matthew F. Warren mfwarren@ncsu.edu

Specialty section: This article was submitted to Animal Nutrition and Metabolism,

a section of the journal

Frontiers in Veterinary Science

Received: 09 January 2020 Accepted: 14 April 2020

Published: 10 June 2020

Citation:

Warren MF, Vu TC, Toomer OT, Fernandez JD and Livingston KA (2020) Efficacy of

1- $\alpha$-Hydroxycholecalciferol Supplementation in Young Broiler Feed Suggests Reducing Calcium

Levels at Grower Phase.

Front. Vet. Sci. 7:245

doi: 10.3389/fvets.2020.00245

\section{Efficacy of}

1- $\alpha$-Hydroxycholecalciferol Supplementation in Young Broiler Feed Suggests Reducing Calcium Levels at Grower Phase

\author{
Matthew F. Warren ${ }^{1 *}$, Thien C. Vu ${ }^{2}$, Ondulla T. Toomer ${ }^{2}$, Juan David Fernandez ${ }^{3}$ and \\ Kimberly A. Livingston ${ }^{1,4}$ \\ 1 Prestage Department of Poultry Science, North Carolina State University, Raleigh, NC, United States, ${ }^{2}$ United States \\ Department of Agriculture, Agricultural Research Service, Raleigh, NC, United States, ${ }^{3}$ Premex, Durham, NC, United States, \\ ${ }^{4}$ Elanco Animal Health, Greenfield, IN, United States
}

Increasing biopotency of cholecalciferol $\left(D_{3}\right)$ from vitamin sources is essential in the poultry industry to meet nutritional demands and counter stressors. $\mathrm{D}_{3}$ exhibits hormonal traits and is responsible for calcium (Ca) absorption. 1- $\alpha$-Hydroxycholecalciferol $(1 \alpha)$ is a synthetic form of $D_{3}$ that has equal efficacy and is cheaper to synthesize than 1,25-dihydroxycholecalciferol (active form of $\mathrm{D}_{3}$ ), on broilers. However, $1 \alpha$ bypasses a critical regulatory point, the kidney, and may consequently lead to toxicity levels of $\mathrm{Ca}$ via Ca absorption. This study examined $1 \alpha$ supplementation in broiler diets with different Ca inclusion levels to determine if $1 \alpha$ at higher Ca levels caused Ca toxicity at starter and grower phases with Ross 708 male broiler chicks. In Experiment 1 (1-15 days of age), chicks were assigned to one of 10 treatment starter diets with five levels of $\mathrm{Ca}$ inclusion $(0.80,0.95,1.10,1.25$, and $1.40 \%)$ with or without $1 \alpha$ supplementation (5 $\mu \mathrm{g}$ $1 \alpha / \mathrm{kg}$ in feed) and eight replicate cages per treatment. In Experiment 2, chicks were fed common starter diet until 16 days of age, and then they were assigned to one of eight treatment diets with four levels of Ca inclusion $(0.54,0.76,0.98$, or $1.20 \%)$ with or without $1 \alpha$ supplementation ( $5 \mu \mathrm{g} 1 \alpha / \mathrm{kg}$ in feed). At the end of both experiments, blood was collected from broilers to determine blood chemistry, including concentrations of vitamin D metabolites. Intestinal tissues were also collected to examine gene expression. In Experiment 1, broilers not fed $1 \alpha$ exhibited a quadratic effect in ionized blood $\mathrm{Ca}$ (iCa) as dietary $\mathrm{Ca}$ inclusion levels increased; $1 \alpha$-fed broilers displayed an increase in iCa as Ca inclusion levels increased ( $\rho=0.0002)$. For Experiment 2, $1 \alpha$-fed broilers displayed a decrease in 25-hydroxycholecalciferol plasma concentration as dietary $\mathrm{Ca}$ inclusion levels increased ( $p=0.035)$; also, increasing Ca inclusion in diets increased expression of duodenal sodium phosphate cotransporter type II b (NPTIlb, $p=0.03$ ). Our findings imply that inclusion of $1 \alpha$ was beneficial because $1 \alpha$ enhanced $\mathrm{Ca}$ absorption during the starter phase; however, to avoid potential Ca toxicity or antagonism while using $1 \alpha$ during the grower phase, there should be consideration with reducing dietary Ca levels.

Keywords: $1-\alpha$-hydroxycholecalciferol, vitamin $D_{3}$, calcium, broiler, blood chemistry, sodium phosphate cotransporter type Ilb, calbindin d28k, 25-hydroxycholecalciferol 


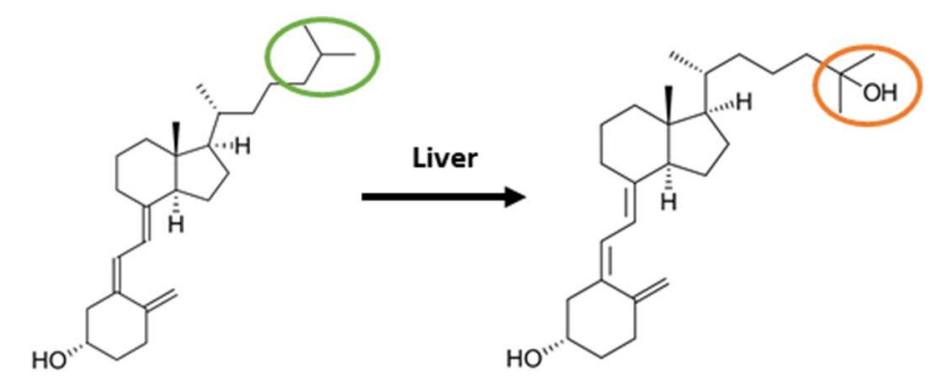

Cholecalciferol $\left(D_{3}\right)$ 25-hydroxycholecalciferol (25-OH-D $\left.\mathrm{D}_{3}\right)$
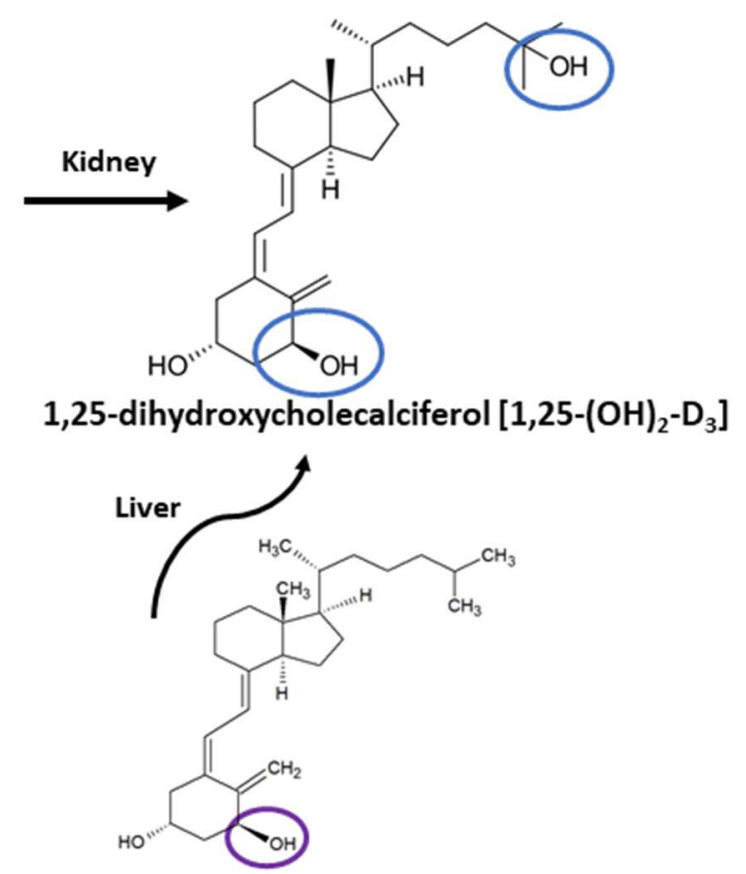

1- $\alpha$-hydroxycholecalciferol $(1 \alpha)$

FIGURE 1 | Metabolic pathway of cholecalciferol (vitamin $\left.D_{3}\right)$ and 1-alpha-hydroxycholecalciferol $(1 \alpha)$ to 1,25 -dihydroxycholecalciferol $\left(1,25-(O H){ }_{2}-D_{3}\right)$. Vitamin $D_{3}$ is converted to 25 -hydroxycholecalciferol $\left(25-\mathrm{OH}-\mathrm{D}_{3}\right)$ in liver, then $25-\mathrm{OH}-\mathrm{D}_{3}$ is converted to $1,25-(\mathrm{OH})_{2} \mathrm{D}_{3}$ in Kidney. $1 \alpha$ travels to liver to be converted to $1,25-(\mathrm{OH})_{2}-\mathrm{D}_{3}$. Green circle highlights $\mathrm{C}-25$ of D3; orange circle highlights $\mathrm{C}-25$ with hydroxyl group for $25-\mathrm{OH}-\mathrm{D}_{3}$; blue circles denote $\mathrm{C}-1$ and $\mathrm{C}-25$ hydroxyl groups of $1,25-(\mathrm{OH})_{2}-\mathrm{D}_{3}$; purple circle highlights $\mathrm{C}-1$ hydroxyl group of $1 \alpha$.

\section{INTRODUCTION}

Vitamin sources with improved bioefficacy are essential in the poultry industry to accommodate nutritional demands of rapidly growing broilers. $\mathrm{D}_{3}$ is necessary for accommodating fast growth of broilers by increasing absorption of calcium (Ca) and its deposition into the bones $(1,2)$. The biopotency of a nutrient can be enhanced by utilizing synthetic forms of the nutrient (3), increasing bioavailability to accommodate a greater response if the nutrient's metabolic effect is dose dependent (4), or adding supplemental enzymes to increase efficacy of the nutrient of interest (5). Supplemental enzymes can be costly, and their effectiveness can vary based on nutrient load and feed processing (6). Synthetic forms of a nutrient could have unintended effects and require further testing (7, 8 ), but they are a viable economical solution for the poultry industry (9).

Previous studies reported inclusion of 1- $\alpha$ hydroxycholecalciferol $(1 \alpha)$, a synthetic analog of vitamin $\mathrm{D}_{3}$, improved $\mathrm{Ca}$ absorption in growing broiler chicks over $\mathrm{D}_{3}$ alone and showed $1 \alpha$ having equal efficacy to 1,25 dihydroxycholecalciferol $\left[1,25-(\mathrm{OH})_{2}-\mathrm{D}_{3}\right](10,11) .1 \alpha$ is cheaper to synthesize and supply in diets compared to $1,25-(\mathrm{OH})_{2}$ $\mathrm{D}_{3}(3,10)$. 1 $\alpha$ 's structure is similar to $1,25-(\mathrm{OH})_{2}-\mathrm{D}_{3}$, but only the 1-alpha carbon is hydroxylated instead of both the 1-alpha and 25-carbon. $1 \alpha$ has greater biopotency over $\mathrm{D}_{3}$ because it is quickly hydroxylated in the liver to its active form, 1,25- $(\mathrm{OH})_{2}-\mathrm{D}_{3}$ (12), and consequently bypasses the hydroxylation step occurring in the kidney. In contrast, $\mathrm{D}_{3}$ requires two hydroxylation steps, first in the liver to form 25-hydroxycholecalciferol $\left(25-\mathrm{OH}-\mathrm{D}_{3}\right)$, which is further hydroxylated in the kidney to $1,25-(\mathrm{OH})_{2}-\mathrm{D}_{3}$ (Figure 1). However, $1 \alpha$ bypasses the critical regulatory hydroxylation by $1 \alpha$-hydroxylase in the kidney $(10,13)$, and $25-\mathrm{OH}-\mathrm{D}_{3}$ levels might significantly increase to result in excessive $\mathrm{Ca}$ absorption leading to hypercalcemia (14). Although levels of ionized blood $\mathrm{Ca}(\mathrm{iCa})$ toxicity in broilers is not established, Hurwitz et al. (15) fed fast-growing chicks diets ranging from 0.4 to $2.0 \%$ Ca with $0.7 \% P$ diets and observed a weight loss in the fast-growing chicks.

$\mathrm{Ca}$ has an important relationship with phosphorus $(P)$, because, together, they comprise a major part of bone structure (16). In the form of limestone or calcium carbonate, $\mathrm{Ca}$ is an inexpensive ingredient and is used as a carrier for many other feed ingredients including mineral premixes and drugs (17). Ca's counterpart, $P$, is one of the more expensive feed ingredients, which limits the amount incorporated into diets. This relationship can result in varying Ca: $P$ ratios from $1: 1$ to 2.6:1 in weight (2). Studies have reported that increasing dietary $\mathrm{Ca}$ levels reduced incidence of tibial dyschondroplasia and therefore improved animal health, welfare, and economic value $(2,18,19)$. Also, an elevated dietary Ca: $P$ ratio of $2.6: 1$ does not appear to negatively affect tibial growth plate morphology at 2 weeks of age (20). 
Although studies have been done on $1 \alpha$ and how it influences vitamin $\mathrm{D}$ status in broilers $(3,21)$, no studies to date have examined how $1 \alpha$ affects $\mathrm{Ca}$ absorption and vitamin $\mathrm{D}$ status when broilers are fed differing levels of dietary Ca. This study explores how $1 \alpha$ impacts vitamin $\mathrm{D}$ status in broiler chickens when they are fed diets with different levels of $\mathrm{Ca}$ in starter and grower phases.

\section{MATERIALS AND METHODS}

Two experiments were conducted to analyze effects of $1 \alpha$ supplementation and increasing levels of $\mathrm{Ca}$ inclusion on blood chemistry of starter and grower phases of broilers. All animal protocols (\# 014-113 for Experiment 1 and \# 17-125-A for Experiment 2) were approved by the Institutional Animal Care and Use Committee at North Carolina State University.

\section{Experiment 1: $1 \alpha$ Supplementation at Starter Phase \\ Birds and Housing}

Four hundred and eighty 1-day-old Ross 708 chicks were hatched at North Carolina State University's Chicken Education Unit in Raleigh, NC. Chicks were housed in Petersime battery cages with six birds per cage and eight replicate cages per treatment. The experimental design was a completely randomized design with or without $1 \alpha$ supplementation (alpha $\mathrm{D}_{3}$, Premex, Antioquia, Colombia) at $5 \mu \mathrm{g} / \mathrm{kg}$ of feed [ $1 \alpha$ dose based on Snow et al. (22), because of its effectiveness] and five levels of Ca inclusion which were added on top of basal diet (Table 1) (23). For this study, broilers from dietary treatments with $1 \alpha$ are noted as $\mathrm{D}_{3}$ $+1 \alpha$; broilers not fed $1 \alpha$ are noted as $D_{3}$. Ca inclusion levels were $0.80,0.95,1.10,1.25$, and $1.40 \%$ with $0.50 \%$ available $P$ in all diets, and birds were fed ad-libitum. The lighting program was set for 23:1 L: D hours for the first 7 days, and the last 8 days of the experiment was set to 17:7. Room temperature was set to be adjusted daily to ensure thermoneutral temperatures as birds grew. Blood was collected at 15 days from two birds per cage via brachial wing vein. At 17 days, all birds were culled. A total of 16 plasma samples per treatment, $D_{3}$ and $D_{3}+1 \alpha$, from broilers given diets at $0.95 \% \mathrm{Ca}$ had their plasma sent (no pooling) to Heartland Assays (Ames, IA) for analysis of vitamin $\mathrm{D}$ metabolites by LC-MS/MS.

\section{Experiment 2: $1 \alpha$ Supplementation at Grower Phase \\ Birds and Housing}

Nine hundred and sixty Ross 708 chicks were hatched at North Carolina State University's Chicken Education Unit in Raleigh, NC. Chicks were housed in 40 floor pens with 24 birds per pen with five replicates per treatment. All chicks were fed a common starter diet (Tables 2, 3) (23) until 17 days of age. It should be noted that all starter diets for these chicks had $1 \alpha$ supplementation (5 $\mu \mathrm{g} / \mathrm{kg}$ of feed). Like Experiment $1,1 \alpha$ used in this experiment was alpha $\mathrm{D}_{3}$ from Premex (Antioquia, Colombia). Like the prior experiment, broilers from dietary treatments with $1 \alpha$ are noted as $D_{3}+1 \alpha$; broilers not fed $1 \alpha$ are noted as $D_{3}$. At 17 days of age, birds were switched to grower diet
TABLE 1 | Ingredient composition and calculated nutrient content of starter basal diet (1-17 days of age) for Ross-708 broilers [From (23)].

\begin{tabular}{|c|c|c|c|}
\hline Ingredient name & $\%$ & Nutrient & $\%$ \\
\hline Corn & 53.25 & Dry matter & 88.55 \\
\hline $\begin{array}{l}\text { Soybean meal, } \\
46 \% \mathrm{CP}\end{array}$ & 30.94 & Moisture & 11.45 \\
\hline Corn gluten meal & 5.00 & Crude protein & 22.71 \\
\hline Poultry fat & 0.00 & Calcium & 0.30 \\
\hline Soybean oil & 4.20 & Total phosphorous & 0.38 \\
\hline Celite & 1.00 & Non-phytate phosphorous & 0.18 \\
\hline $\begin{array}{l}\text { Filler (Limestone + } \\
\mathrm{CaHPO}_{4}+\text { Sand) }\end{array}$ & 3.15 & Phytate phosphorous & 0.24 \\
\hline Salt $(\mathrm{NaCl})$ & 0.29 & Total methionine & 0.67 \\
\hline $\begin{array}{l}\text { DL-Methionine, } \\
\text { 99\% }\end{array}$ & 0.30 & Total cysteine & 0.36 \\
\hline $\begin{array}{l}\text { Sodium } \\
\text { bicarbonate }\end{array}$ & 0.31 & Total lysine & 1.39 \\
\hline $\begin{array}{l}\text { Dicalcium } \\
\text { phosphate } \\
\left(\mathrm{CaHPO}_{4}\right)^{\mathrm{a}}\end{array}$ & 0.27 & Total tryptophan & 0.25 \\
\hline Mineral premix ${ }^{b}$ & 0.20 & Total threonine & 0.98 \\
\hline $\begin{array}{l}\text { Limestone Cerne } \\
\text { Pure Cal } 12-40^{\circ}\end{array}$ & 0.17 & Total isoleucine & 0.94 \\
\hline $\begin{array}{l}\text { L-Lysine-HCl, } \\
78.8 \%\end{array}$ & 0.38 & Total valine & 1.05 \\
\hline $\begin{array}{l}\text { Choline chloride, } \\
60 \% \text { choline }\end{array}$ & 0.18 & Total leucine & 2.13 \\
\hline L-Threonine, 98\% & 0.15 & Total arginine & 1.40 \\
\hline Selenium premix & 0.05 & Total sulfur amino acids & 1.04 \\
\hline Vitamin premix ${ }^{d}$ & 0.10 & Total glycine & 0.88 \\
\hline Anticoccidiale ${ }^{e}$ & 0.05 & Sodium & 0.22 \\
\hline Natuphos $E^{\circledR f}$ & 0.01 & Potassium & 0.85 \\
\hline \multirow[t]{13}{*}{ Total } & 100.00 & Chloride & 0.29 \\
\hline & & Digestible lysine & 1.28 \\
\hline & & Digestible methionine & 0.63 \\
\hline & & Digestible cysteine & 0.31 \\
\hline & & $\begin{array}{l}\text { Digestible total sulfur amino } \\
\text { acids }\end{array}$ & 0.94 \\
\hline & & Digestible threonine & 0.85 \\
\hline & & Digestible tryptophan & 0.22 \\
\hline & & Digestible isoleucine & 0.84 \\
\hline & & Digestible leucine & 2.04 \\
\hline & & Digestible valine & 0.95 \\
\hline & & Digestible arginine & 1.30 \\
\hline & & $\begin{array}{l}\text { Metabolizable Energy, } \\
\mathrm{kcal} / \mathrm{kg}\end{array}$ & 3,000 \\
\hline & & $\begin{array}{l}\text { Dietary electrolyte balance, } \\
\mathrm{mEq} / 100 \mathrm{~g}\end{array}$ & 254 \\
\hline
\end{tabular}

${ }^{a}$ Dicalcium phosphate contains $19.79 \%$ calcium, $17.91 \%$ phosphorus, and $17.73 \%$ available phosphorus.

${ }^{b}$ Trace minerals provided per kg of premix: $60 \mathrm{~g}$ manganese $\left(\mathrm{Mn} \mathrm{SO}^{4}\right) ; 60 \mathrm{~g}$ zinc $\left(\mathrm{ZnSO}{ }^{4}\right)$; $40 \mathrm{~g}$ iron (FeSO4); $5 \mathrm{~g}$ copper $\left(\mathrm{CuSO}^{4}\right) ; 1.25 \mathrm{~g}$ iodine $\left[\mathrm{Ca}\left(\mathrm{IO}^{3}\right)^{2}\right]$.

${ }^{c}$ Limestone (Cerne Pure Cal 12-4) contains $39.467 \%$ calcium.

${ }^{d}$ Vitamins provided per $\mathrm{kg}$ of premix: 13,227,513 IU vitamin A; 3,968,253 IU vitamin $D^{3} ; 66,137$ IU vitamin $E ; 39.6 \mathrm{mg}$ vitamin B12; 13,227 mg riboflavin; 110,229 mg niacin; 22,045 mg d-pantothenic acid; 3,968 mg menadione; 2,204 mg folic acid; 7,936 mg vitamin B6; 3,968 mg thiamine; $253.5 \mathrm{mg}$ biotin.

${ }^{e}$ Coban ${ }^{\circledR} 90$ (Monensin), Elanco Animal Health, Greenfield, IN, at $500 \mathrm{~g} /$ ton in the starter and grower diets.

${ }^{f}$ Natuphos $E^{\circledR}$ (500 FTU/kg, 50 g/ton FTU). 
TABLE 2 | Ingredient composition of starter diet and grower basal diets for Ross-708 male broilers [From (23)].

\begin{tabular}{lll}
\hline Ingredient name & Starter (1-16 d) $\quad$ Grower (17-35 d) \\
\hline
\end{tabular}

(\%)

\begin{tabular}{|c|c|c|}
\hline Corn & 55.84 & 57.59 \\
\hline Soybean meal, $46 \%$ CP & 31.70 & 26.89 \\
\hline Corn gluten meal & 4.90 & 5.00 \\
\hline Soybean oil & 3.12 & 4.85 \\
\hline Dicalcium phosphate $\left(\mathrm{CaHPO}_{4}\right)^{\mathrm{a}}$ & 1.37 & 0.31 \\
\hline Limestone Cerne Pure Cal $12-40^{\mathrm{b}}$ & 1.06 & 0.01 \\
\hline Sodium bicarbonate & 0.36 & 0.23 \\
\hline L-Lysine-HCl, 78.8\% & 0.36 & 0.31 \\
\hline DL-Methionine, 99\% & 0.29 & 0.25 \\
\hline Salt (NaCl) & 0.28 & 0.28 \\
\hline Mineral premix ${ }^{c}$ & 0.20 & 0.20 \\
\hline Choline chloride, $60 \%$ & 0.18 & 0.18 \\
\hline L-threonine & 0.13 & 0.10 \\
\hline Vitamin premix ${ }^{d}$ & 0.10 & 0.10 \\
\hline Anticoccidial $^{\mathrm{e}}$ & 0.05 & 0.05 \\
\hline Selenium premix & 0.05 & 0.05 \\
\hline Sand & 0.01 & 3.60 \\
\hline Celite & - & 1.00 \\
\hline Limestone dicalcium base & - & 2.60 \\
\hline $1 \alpha(\mathrm{OH}) \mathrm{D}_{3}$ & 0.00125 & - \\
\hline Natuphos $E^{\circledR f}$ & 0.005 & 0.005 \\
\hline Total & 100.00 & 100.00 \\
\hline
\end{tabular}

${ }^{a}$ Dicalcium phosphate contains $19.79 \%$ calcium, $17.9091 \%$ phosphorus, and $17.73 \%$ available phosphorus (99\%).

${ }^{b}$ Limestone (Cerne Pure Cal 12-4) contains 39.01\% calcium.

${ }^{c}$ Trace minerals provided per $\mathrm{kg}$ of premix: $60 \mathrm{~g}$ manganese $\left(\mathrm{Mn} \mathrm{SO}^{4}\right) ; 60 \mathrm{~g}$ zinc $\left(\mathrm{ZnSO}{ }^{4}\right)$; $40 \mathrm{~g}$ iron (FeSO4); $5 \mathrm{~g}$ copper $\left(\mathrm{CuSO}{ }^{4}\right) ; 1.25 \mathrm{~g}$ iodine $\left[\mathrm{Ca}\left(\mathrm{IO}^{3}\right)^{2}\right]$.

${ }^{d}$ Vitamins provided per $\mathrm{kg}$ of premix: 13,227,513 U vitamin A; 3,968,253 IU vitamin $D^{3} ; 66,137 \mathrm{IU}$ vitamin E; $39.6 \mathrm{mg}$ vitamin B12; 13,227 mg riboflavin; 110,229 mg niacin; 22,045 mg d-pantothenic acid; 3,968 mg menadione; 2,204 mg folic acid; 7,936 mg vitamin B6; 3,968 mg thiamine; $253.5 \mathrm{mg}$ biotin.

${ }^{e}$ Coban ${ }^{\circledR} 90$ (Monensin), Elanco Animal Health, Greenfield, IN, at $500 \mathrm{~g} /$ ton in the starter and grower diets.

${ }^{f}$ Natuphos $E^{\circledR}$ (500 FTU/kg, 50 g/ton FTU).

and assigned to one of eight treatment groups with four levels of Ca inclusion (added on top of basal diet; 0.54, 0.76, 0.98, or $1.20 \%$ of diet) and with or without $1 \alpha$ supplementation $(5 \mu \mathrm{g} / \mathrm{kg}$ of feed) with five replicate pens per treatment. All diets contained $0.50 \%$ available $P$. At 35 days of age, blood was collected from two birds per pen and euthanized, and duodenal and jejunal tissues were collected. Duodenal tissue was washed with saline and stored in RNAlater at $-20^{\circ} \mathrm{C}$. Jejunal tissue was washed with saline and stored in $4 \%$ formalin for histology. Plasma from each treatment was pooled using four birds per housing row into one pooled sample, for a total of three pooled reps per treatment and sent to Heartland Assays (Ames, IA) for analysis of vitamin $\mathrm{D}$ metabolites.

\section{Blood Collection and Blood Chemistry}

Blood was collected into BD Vacutainer lithium heparin tubes (Franklin Lakes, NJ) from two birds per cage (Experiment 1) or

TABLE 3 | Nutritional content of basal starter and grower diets for Ross-708 male broilers [From (23)].

Nutrient $\quad$ Starter (1-16 d) Grower (17-35 d)

Nutrient

$(\%)$

\begin{tabular}{|c|c|c|}
\hline Crude protein & 23.14 & 20.94 \\
\hline Calcium & 0.87 & 0.24 \\
\hline Total phosphorous & 0.58 & 0.36 \\
\hline Non-phytate phosphorous & 0.38 & 0.18 \\
\hline Phytate phosphorous & 0.24 & 0.22 \\
\hline Total methionine & 0.65 & 0.59 \\
\hline Total cysteine & 0.38 & 0.35 \\
\hline Total lysine & 1.41 & 1.23 \\
\hline Total tryptophan & 0.27 & 0.24 \\
\hline Total threonine & 0.97 & 0.86 \\
\hline Total isoleucine & 0.97 & 0.87 \\
\hline Total valine & 1.09 & 0.98 \\
\hline Total leucine & 2.15 & 2.00 \\
\hline Total arginine & 1.43 & 1.27 \\
\hline Total sulfur amino acids & 1.03 & 0.93 \\
\hline Total glycine & 0.90 & 0.81 \\
\hline Digestible lysine & 1.28 & 1.12 \\
\hline Digestible methionine & 0.63 & 0.56 \\
\hline Digestible cysteine & 0.32 & 0.29 \\
\hline Digestible total sulfur amino acids & 0.94 & 0.85 \\
\hline Digestible threonine & 0.85 & 0.75 \\
\hline Digestible tryptophan & 0.23 & 0.21 \\
\hline Digestible, isoleucine & 0.88 & 0.79 \\
\hline Digestible leucine & 1.99 & 1.85 \\
\hline Digestible valine & 0.96 & 0.87 \\
\hline Digestible arginine & 1.33 & 1.18 \\
\hline Sodium & 0.23 & 0.19 \\
\hline Potassium & 0.88 & 0.781 \\
\hline Chloride & 0.28 & 0.275 \\
\hline Metabolizable energy, kcal/kg & $3,000.00$ & $3,100.00$ \\
\hline Dietary electrolyte balance, mEq/kg & 267.53 & 227.10 \\
\hline
\end{tabular}

pen (Experiment 2). Blood chemistry parameters and iCa were determined using an $\mathrm{i}-\mathrm{STAT}^{\mathrm{TM}}$ blood analyzer (Abaxis, Union City, CA) using CG8+ cartridges (Abaxis, Union City, CA), and the remaining blood was spun down, and plasma was collected and stored at $-80^{\circ} \mathrm{C}$ to determine vitamin $\mathrm{D}$ metabolite levels. All plasma samples were sent to Heartland Assays (Ames, IA) for measuring $\mathrm{D}_{3}, 25-\mathrm{OH}-\mathrm{D}_{3}$, and 24,25-dihydroxycholecalciferol $\left[24,25-(\mathrm{OH})_{2}-\mathrm{D}_{3}\right.$, the inactive form of $\left.\mathrm{D}_{3}\right]$ by LC-MS/MS.

\section{RNA Extraction and qPCR}

Total mRNA was extracted from duodenal tissue using Qiagen's RNeasy Mini Kit (Germantown, MD). Extracted RNA was diluted and normalized to $\sim 200 \mathrm{ng} / \mu \mathrm{l}$ and reverse transcribed to complementary DNA (cDNA) using Applied Biosystems' High-Capacity cDNA Reverse Transcription Kit (Thermo Fisher Scientific, Waltham, MA) and protocol to make a $20-\mu l$ working solution. Cycling procedure for reverse transcription started with 
TABLE 4 | Primer sequences for quantitative real-time PCR (qPCR).

\begin{tabular}{|c|c|c|c|c|}
\hline Gene & Orientation & $\begin{array}{l}\text { Primer sequence } \\
\left(5^{\prime}-3^{\prime}\right)\end{array}$ & $\begin{array}{l}\text { Size } \\
\text { (bp) }\end{array}$ & Accession \# \\
\hline \multirow[t]{2}{*}{ VDR } & Forward & $\begin{array}{l}\text { TGCCTCCAGTCTGGC } \\
\text { ATCTC }\end{array}$ & 297 & NM_205098.1 \\
\hline & Reverse & $\begin{array}{l}\text { GGTGATITTGCAGTC } \\
\text { CCCGT }\end{array}$ & & \\
\hline \multirow[t]{2}{*}{ MUC2 } & Forward & $\begin{array}{l}\text { GTGTGCCCTGATGTC } \\
\text { ACAGA }\end{array}$ & 246 & XM_015286749.1 \\
\hline & Reverse & $\begin{array}{l}\text { GGCCTGAGCCTTGGT } \\
\text { ACATT }\end{array}$ & & \\
\hline \multirow[t]{2}{*}{ CALB } & Forward & $\begin{array}{l}\text { TTGCCGACGGAG } \\
\text { GAGAATाT }\end{array}$ & 261 & NM_205513.1 \\
\hline & Reverse & $\begin{array}{l}\text { GGCCAGTTCAGTAAG } \\
\text { CTCCA }\end{array}$ & & \\
\hline \multirow[t]{2}{*}{ NPTIllb } & Forward & $\begin{array}{l}\text { AACTGGCTTGCTGTG } \\
\text { ताTC }\end{array}$ & 423 & NM_204474.2 \\
\hline & Reverse & $\begin{array}{l}\text { GATGGCAAGATCAGG } \\
\text { CAGGT }\end{array}$ & & \\
\hline \multirow[t]{2}{*}{ GAPDH } & Forward & $\begin{array}{l}\text { TGTTGTTGACCTGAC } \\
\text { CTGCC }\end{array}$ & 291 & NM_204305.1 \\
\hline & Reverse & $\begin{array}{l}\text { CTGGCTCACTCCTTG } \\
\text { GATGC }\end{array}$ & & \\
\hline
\end{tabular}

$25^{\circ} \mathrm{C}$ for $10 \mathrm{~min}, 37^{\circ} \mathrm{C}$ for $120 \mathrm{~min}$, and $85^{\circ} \mathrm{C}$ for $5 \mathrm{~min}$ and then held at $5^{\circ} \mathrm{C}$ indefinitely until storage or use.

Genes expressed for qPCR were vitamin D receptor (VDR) mucin 2 (MUC2), calbindin D28k (CALB), sodium phosphate cotransporter type IIb (NPTIIb), and glyceraldehyde (GAPDH) as a housekeeping gene (Table 4). qPCR was conducted using PowerUP SYBR Master Mix (Life Technologies, Grand Island, NY) using the Applied Biosystems protocol to make a 20- $\mu$ l working solution and using the Applied Biosystems StepOnePlus Real-Time PCR System (Carlsbad, CA). Cycling procedure started with $95^{\circ} \mathrm{C}$ for $10 \mathrm{~min}$ and then 40 cycles of $95^{\circ} \mathrm{C}$ for $15 \mathrm{~s}$ for denaturing and $15 \mathrm{~s}$ at $60^{\circ} \mathrm{C}$ for annealing. All samples were run in triplicates.

\section{Histology}

Light microscopy $(40 \times$ magnification) was used for morphometric analysis of histological serial sections of jejuna prepared using standard hematoxylin and eosin staining to examine if dietary treatments affected gut morphology. Villus height, crypt depth, and villus width were measured using image analyzer AmScope version 3.7 (Irvine, CA). Ten measurements for villus surface area $\left(\mu \mathrm{m}^{2}\right)$ and villus height/crypt depth were made per experimental unit (bird).

\section{Data Analysis}

All data are reported as mean \pm standard error of the mean. Statistical analyses were conducted using general linear model using the following model:

$$
Y_{\mathrm{ijk}}=\mu+\beta_{1}(\mathrm{Ca})_{\mathrm{i}}+\beta_{2}(\mathrm{Vit} . \mathrm{D})_{\mathrm{j}}+\beta_{3}(\mathrm{Ca} \times \mathrm{Vit} . \mathrm{D})_{\mathrm{ij}}+\varepsilon_{\mathrm{ijk}}
$$

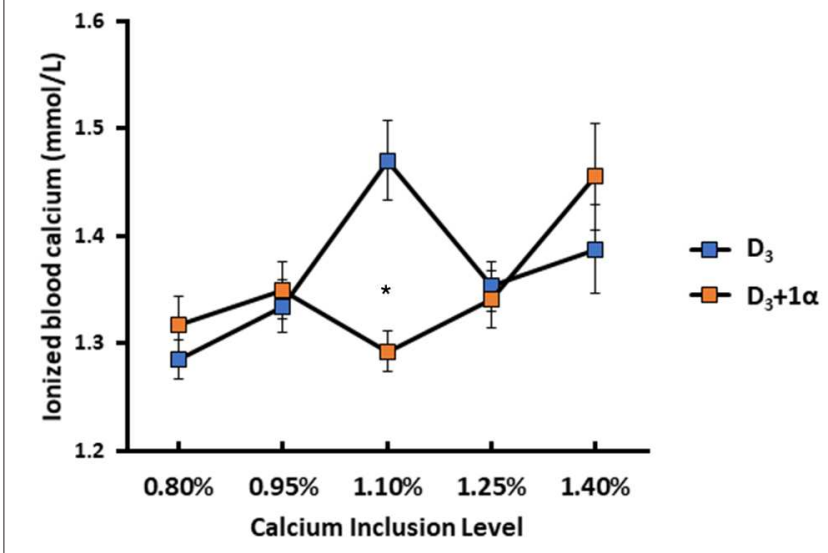

FIGURE 2 | lonized blood calcium of $15 \mathrm{~d}$ broiler chickens fed different levels of calcium with or without 1-alpha hydroxycholecalciferol supplementation $\left(D_{3}\right.$ $+1 \alpha ; D_{3}$, respectively). Line graphs show means \pm standard error means $(n=$ 8). Interaction effect observed between calcium inclusion and $1 \alpha$ supplementation [General linear model (GLM), ${ }^{\star} p<0.05$ ].

where $\mathrm{Y}_{\mathrm{ijk}}$ is the individual observation; $\mu$ is the experimental mean; $\mathrm{Ca}$ is the $\mathrm{Ca}$ inclusion effect of the ith level; Vit. D is the effect of $1 \alpha$ supplementation of the $j$ th level; and $\varepsilon_{i j k}$ is the error. $\beta$ s are the slopes for each predictor. The interaction term of $\mathrm{Ca} \times$ Vit. $\mathrm{D}$ is also included in the model. This model was used to compare differences in blood chemistry concentrations, vitamin D metabolite plasma concentrations, and relative gene expression using SAS $9.4^{\circledR}$. The Tukey-Kramer test was used for multiple comparisons for differences between and within treatment groups for blood chemistry, vitamin D metabolites, and mRNA relative expression. All mRNA relative expressions were normalized using $2^{-\Delta \Delta C T}$ (24) with GAPDH as a housekeeping gene control. Statistical significance was established at $p<0.05$, and statistical trends were noted when $0.05<p \leq 0.10$.

\section{RESULTS}

\section{Experiment 1: Starter Diet}

\section{$1 \alpha$ Efficacy Dependent on Dietary Calcium Inclusion} Levels With iCa Concentration

In terms of blood chemistry, only iCa exhibited any differences between $0.80 \%$ and $1.10 \% \mathrm{Ca}$ inclusion (Figure 2). For $\mathrm{D}_{3}+1 \alpha$ broilers, iCa increased as dietary $\mathrm{Ca}$ increased $(1.46 \mathrm{mmol} / \mathrm{L} \mathrm{iCa}$ at $1.40 \% \mathrm{Ca}$ inclusion). Broilers from $\mathrm{D}_{3}$ treatments exhibited a quadratic effect with increasing iCa from $1.29 \mathrm{mmol} / \mathrm{L}$ to a peak of $1.47 \mathrm{mmol} / \mathrm{L}$ when dietary $\mathrm{Ca}$ increased from 0.80 to $1.10 \%$ $\mathrm{Ca}$ inclusion. Once dietary $\mathrm{Ca}$ went over $1.10 \%$, iCa decreased $(p=0.006)$.

\section{$1 \alpha$ Supplementation Did Not Affect Plasma Vitamin $\mathrm{D}_{3}$ Levels at $0.95 \% \mathrm{Ca}$ Inclusion}

At $0.95 \% \mathrm{Ca}$ inclusion, $1 \alpha$ supplementation did not affect plasma concentrations of $24,25-(\mathrm{OH})_{2}-\mathrm{D}_{3}$ or $25-\mathrm{OH}-\mathrm{D}_{3}$. A statistical trend was observed with $\mathrm{D}_{3}+1 \alpha$ broilers having 
A

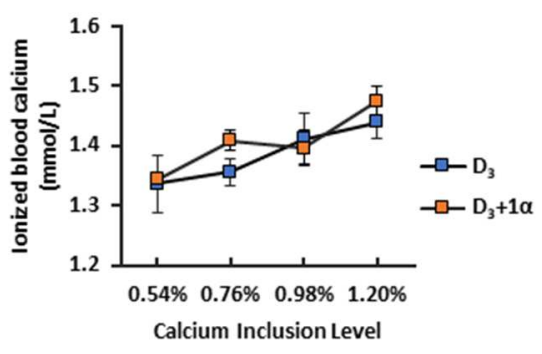

C

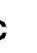

B

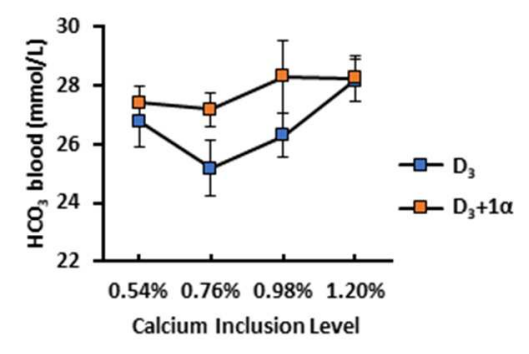

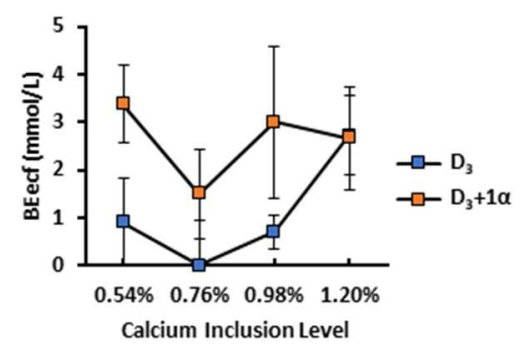

FIGURE 3 | Selected blood chemistry of 35 d broiler chickens fed different levels of calcium with or without 1 -alpha-hydroxycholecalciferol supplementation ( $\mathrm{D}_{3}+1 \alpha$; $\mathrm{D}_{3}$, respectively). (A) lonized blood calcium (B) Base excess of extracellular fluid (BEecf) (C) Blood bicarbonale ( $\left.\mathrm{HCO}_{3}\right)$. Line graphs show means \pm standard error means $(n=5)$. Calcium inclusion effect observed for ionized blood calcium; $1 \alpha$ supplementation effect with BEect [General linear models (GLM), $p<0.05]$.

a higher $\mathrm{D}_{3}$ concentration compared to $\mathrm{D}_{3}$ broilers (data not shown; $p=0.07$ ).

\section{Experiment 2: Grower Diet Specific Blood Chemistry Parameters Are Affected by Ca Inclusion or $1 \alpha$ Supplementation}

$1 \alpha$ had no effect on 35 -days broilers' iCa concentration, but when $1 \alpha$ was not included in the diet, iCa concentration increased as dietary $\mathrm{Ca}$ inclusion level increased ( $\mathrm{Ca}$ inclusion: $p=0.003$; Figure $3 \mathrm{~A}) \cdot \mathrm{D}_{3}+1 \alpha$ broilers had higher blood bicarbonate concentration ( $p=0.041$ ), and a trend was observed with bicarbonate concentration with increasing $\mathrm{Ca}$ inclusion levels ( $p=0.08$; Figure 3B). Base excess of extracellular fluid (BEecf) is when hydrogen ions diffuse into red blood cells which causes plasma alkalinity to rise as a result as a base excess (25). The statistical model for BEecf initially exhibited a statistical trend with both vitamin D and Ca inclusion. However, the model expresses a statistical difference when only vitamin $\mathrm{D}$ is in the model, which is reported in this study. BEecf concentration was increased in $\mathrm{D}_{3}+1 \alpha$ broilers $(p=0.03$; Figure 3C).

\section{Plasma Vitamin $\mathrm{D}_{3}$ Metabolite Concentration Is Affected by $1 \alpha$ Supplementation and Calcium Inclusion}

No difference was noted among dietary treatments with $24,25-(\mathrm{OH})_{2}-\mathrm{D}_{3}$ plasma concentration ( $p=0.19$; Figure 4A). Broilers fed $1 \alpha$ supplementation had a decrease in plasma
25-OH-D 3 concentration $(p<0.0001$; Figure 4B). There was no dietary $\mathrm{Ca}$ inclusion effect on plasma $25-\mathrm{OH}-$ $\mathrm{D}_{3}$ concentration. Broilers fed $1 \alpha$ had a linear decrease in plasma $\mathrm{D}_{3}$ as calcium inclusion levels increased, except that broilers from $0.76 \%$ Ca treatment had the highest $D_{3}$ concentration ( $p=0.02$; Figure $4 \mathrm{C}$ ). Relative concentrations of each measured vitamin $\mathrm{D}_{3}$ metabolite were shown to illustrate how $1 \alpha$ supplementation affected vitamin $\mathrm{D}_{3}$ metabolites (Figure 4D).

\section{Higher Calcium Inclusion Levels Influenced Duodenal NPTIlb Gene Expression}

No Ca inclusion effects or $1 \alpha$ supplementation effects were observed for CALB ( $p=0.52$; Figure 5A). Broilers in $\mathrm{D}_{3}+1 \alpha$ treatments had decreased relative expression of MUC2 compared to control treatment ( $p=0.002$; Figure 5B). A statistical trend was denoted for VDR expression for $1 \alpha$ supplementation $(p=$ $0.084)$ and $\mathrm{Ca}$ inclusion $(p=0.055)$ with a slight increase in expression with $\mathrm{D}_{3}$ broilers, but a larger sample size may be necessary to observe an effect (Figure $5 \mathrm{C}$ ). $\mathrm{D}_{3}$ broilers expressed an increase in NPTIIb expression compared to $\mathrm{D}_{3}+1 \alpha$ broilers as dietary Ca increased ( $p=0.03$, Figure 5D).

\section{$1 \alpha$ Supplementation and Calcium Inclusion Level Did Not Affect Height/Crypt Depth Ratio of Jejunal Villi}

Feeding $1 \alpha$ to broilers had no impact on surface area or height/crypt depth ratio of villi (data not shown; $p=0.45$ or $p$ $=0.86$; respectively). Similarly, Ca inclusion levels did not affect 
$\mathbf{A}$

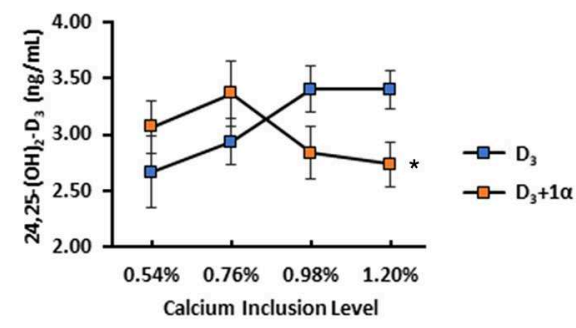

C

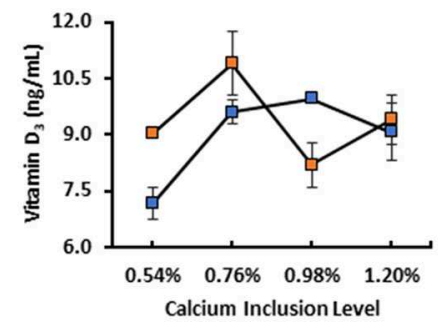

$\mathbf{B}$

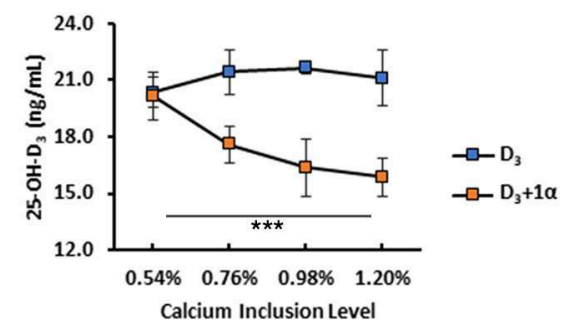

D

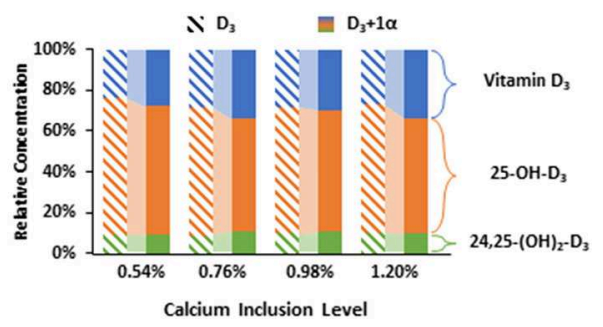

FIGURE 4 | Vitamin D metabolite plasma concentrations of $35 \mathrm{~d}$ broiler chickens fed different levels of calcium with or without 1-alpha-hydroxycholecalciferol supplementation $\left(\mathrm{D}_{3}+1 \alpha ; \mathrm{D}_{3}\right.$, respectively). (A) 24,25-dihydroxycholecalciferol $\left(24,25-(\mathrm{OH})_{2}-\mathrm{D}_{3}\right)$ (B) 25 -hydroxycholecalciferol $\left(25-\mathrm{OH}-\mathrm{D}_{3}\right)$ (C) Cholecalciferol $(\mathrm{Vitamin}$ $D_{3}$ ) (D) Comparison of relative concentration between each vitamin $D_{3}$ metabolite between $D_{3}$ and $D_{3}+1 \alpha$ groups; diagonal patterned bars denote $D_{3}$ and solid bars denote $\mathrm{D}_{3}+1 \alpha .1 \alpha$ supplementation effect with $25-\mathrm{OH}-\mathrm{D}_{3}$. Interaction between calcium inclusion and $1 \alpha$ supplementation with plasma vitamin $\mathrm{D}_{3}$. Line graphs show means \pm standard error means $\left[n=3\right.$; General linear models (GLM), $\left.{ }^{*} p<0.05 ;{ }^{* \star *} p \leq 0.0001\right]$.

A

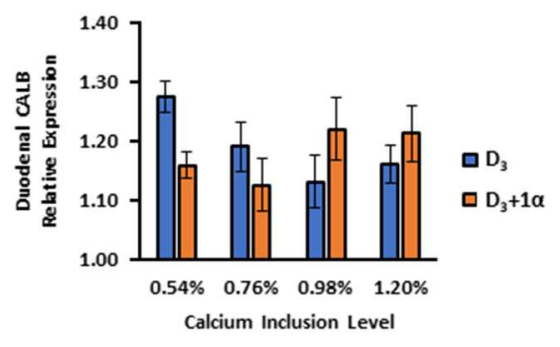

C

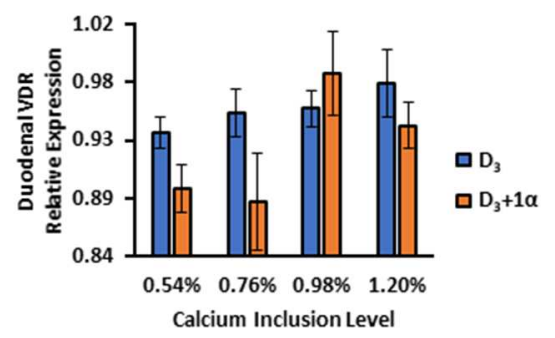

B

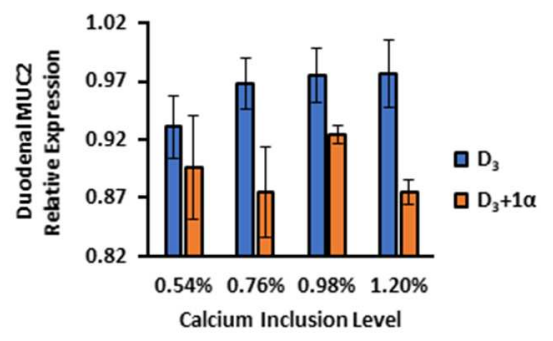

D

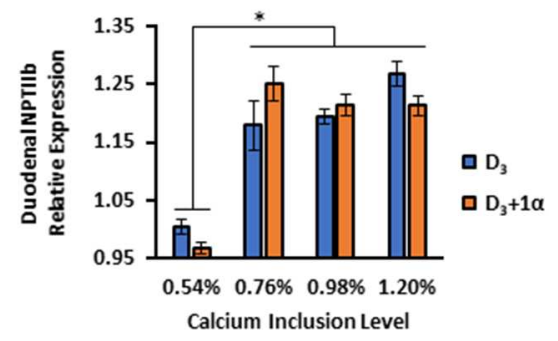

FIGURE 5 | Relative gene expression in duodenal tissue of $35 \mathrm{~d}$ broiler chickens fed different levels of calcium with or without 1-alpha-hydroxycholecalciferol supplementation ( $D_{3}+1 \alpha ; D_{3}$, respectively). (A) Calbindin d28k (CALB) (B) Mucin 2 (MUC2) (C) Vitamin D receptor (VDR) (D) Sodium-phosphate cotransporter type II b (NPTIllb). Duodenal tissue was analyzed using qPCR normalized against glyceraldehyde phosphate dehydrogenase (GAPDH; housekeeping gene) expression ( $n=$ 5). [General linear models (GLM). * $p<0.05]$. 
either parameter $(p=0.40$ and $p=0.34$ for surface area and height/crypt depth ratio, respectively).

\section{DISCUSSION}

Our study indicated that including $1 \alpha$ in young broiler diets can improve their blood iCa status during the starter phase with increased $\mathrm{Ca}$ inclusion. $1 \alpha$ supplementation at the grower phase can also cause a decrease in plasma vitamin D metabolites in young broilers. $1 \alpha$ supplementation seems to exhibit greater efficacy during the starter phase compared to the grower phase of broiler diets in its alteration of blood iCa. However, broilers fed grower diets with $1 \alpha$ supplementation had a decrease in plasma $25-\mathrm{OH}-\mathrm{D}_{3}$ concentration as $\mathrm{Ca}$ inclusion increased. An interesting effect of $1 \alpha$ supplementation on blood chemistry was that it increased BEecf concentration during the grower phase. Increased BEecf concentration indicated broilers were metabolically exceeding their capacity to maintain acid-base balance in body, resulting in more alkali blood, an effect previously described by Mongin (26). We argue that this effect is caused by excess dietary $\mathrm{Ca}$ which has electrolytical potential as a cation when present in tissues (27), which has biological implications for potentially using $1 \alpha$ in diets with lower $\mathrm{Ca}$ levels for the grower phase; otherwise, these birds are under some form of duress to reduce BEecf to homeostatic balance.

Blood $\mathrm{Ca}$ is tightly regulated for various reasons including the need to control Ca distribution into tissues and to maintain blood $\mathrm{pH}$ (28). Levels of $\mathrm{iCa}$ in our two experiments were similar in range to those studies which reported plasma $\mathrm{Ca}$ concentrations from young broilers of similar ages $(21,29,30)$, indicating no unusual physiological levels to denote Ca deficiency or toxicity. For broilers from the starter diet experiment, at 1.10\% Ca inclusion, $\mathrm{D}_{3}+1 \alpha$ broilers had decreased iCa compared to $\mathrm{D}_{3}$ broilers; this exhibits $1 \alpha$ 's increased efficacy on Ca utilization because $\mathrm{D}_{3}+1 \alpha$ broilers most likely absorbed $\mathrm{Ca}$ for bone metabolism compared to $\mathrm{D}_{3}$ broilers having a much higher blood iCa concentration. Han et al. (21) fed broiler chicks different levels of $1 \alpha$ and reported a linear effect with increasing $1 \alpha$ also increased plasma $\mathrm{Ca}$ concentration, although their broilers had a higher average concentration compared to our birds by almost $1 \mathrm{mmol} / \mathrm{L}$ with their $5 \mu \mathrm{g} / \mathrm{kg} 1 \alpha$ supplementation with $0.25 \% \mathrm{Ca}$ diet. However, their plasma $\mathrm{Ca}$ was collected at 21 days compared to our study, which collected $\mathrm{iCa}$ at 15 days. Growth performance of these birds had a quadratic relationship with $\mathrm{Ca}$ inclusion and increased $\mathrm{Ca}$ digestibility with chicks fed $1 \alpha$ relative to Ca inclusion levels (23). Further investigation with an even lower dietary Ca inclusion level may help fit a better regression line for this relationship with $1 \alpha$ 's efficacy on $\mathrm{Ca}$ absorption into bone. Examining hydroxylase expression in kidney and liver tissues could determine whether pathological issues are developing due to $\mathrm{Ca}$ intake. There would be greater expression of 25-hydroxylase in liver with regard to $1.40 \% \mathrm{Ca}$ inclusion level of $\mathrm{D}_{3}+1 \alpha$ broilers having a higher iCa concentration compared to their counterparts without $1 \alpha$ supplementation. If this case were to occur, then histopathological examination of kidneys and liver would verify soft-tissue calcification.

When broilers were fed at the grower phase, increasing $\mathrm{Ca}$ inclusion levels caused an increase in blood Ca concentration at the grower phase, regardless of $1 \alpha$ supplementation. Also, both $\mathrm{Ca}$ inclusion levels and $1 \alpha$ did not affect body weight of broilers fed at the grower phase (23). Our result is in agreement with that of Sebastian et al. (31) who also report how increasing $\mathrm{Ca}$ inclusion levels led to increased blood Ca concentration. Increased blood $\mathrm{Ca}$ concentration indicates that $\mathrm{Ca}$ requirements are met in these chickens and that $\mathrm{Ca}$ is most likely being excreted or stored in bone, which was observed with various strains of broilers because of how tightly regulated blood Ca is $(29,31)$. It should also be noted that $1 \alpha$ caused birds to have a consistent $\mathrm{Ca}$ digestibility, whereas birds not fed $1 \alpha$ had a negative quadratic relationship with $\mathrm{Ca}$ digestibility as Ca inclusion increased (23). $1 \alpha$ 's impact on increasing blood bicarbonate concentration is probably caused by a shift in acid-base balance. We did not observe any changes in $\mathrm{pH}$ or $\mathrm{CO}_{2}$ blood concentration; however, we speculate bicarbonate's increase without any change to $\mathrm{CO}_{2}$ implies these broilers may be under physiological stress with alkalosis. Metabolic alkalosis can be caused by chloride depletion but can also be caused by hypercalcemia because hypercalcemia increases bicarbonate resorption (32). Therefore, it is possible that $1 \alpha$, with addition of dietary vitamin $\mathrm{D}$, could have caused greater $\mathrm{Ca}$ absorption that led to greater bicarbonate being resorbed. There is also the possibility that at the time of blood sampling, the broilers may have metabolically compensated for the partial pressure of $\mathrm{CO}_{2}$ to maintain $\mathrm{pH}$ in the reaction to increased bicarbonate levels (33). A caveat of our study is that we did not examine blood chloride levels, which could potentially explain the BEecf increase. Future studies should explore the mechanism with $\mathrm{Ca}$ inclusion in broilers on why blood bicarbonate and BEecf increased even though $\mathrm{pH}$ did not change.

There were no differences in vitamin $\mathrm{D}_{3}$ metabolite blood concentrations in broiler chicks fed starter diets with or without $1 \alpha$ supplementation at $0.95 \%$ Ca inclusion. $1 \alpha$ may not be affecting vitamin $\mathrm{D}$ pathways considering there was no difference in blood iCa concentration at this level. Based on data from the National Research Council (34), 0.95\% Ca inclusion is closest to the starter phase minimum requirements for broilers. We may find a difference in vitamin D metabolite blood concentrations in broilers fed $1.10 \% \mathrm{Ca}$ because of how drastically iCa concentration differed between $\mathrm{D}_{3}$ and $\mathrm{D}_{3}+$ $\alpha$ broilers.

However, $1 \alpha$ 's effects are more pronounced when broilers are older and fed a grower diet. An inverse relationship of vitamin $\mathrm{D}_{3}$ plasma concentration implies how $1 \alpha$ 's influence in vitamin $\mathrm{D}$ metabolism is dependent on dietary $\mathrm{Ca}$ inclusion (Figure 4C). This relationship is also connected to $25-\mathrm{OH}-\mathrm{D}_{3}$ because $1 \alpha$ is likely exerting an effect in order to reduce vitamin $\mathrm{D}_{3}$ because of $1 \alpha$ 's efficacy. We believe $\mathrm{D}_{3}$ is being excreted in feces, but we are unable to find any poultry-related studies that report vitamin D in excreta. A human study discussed how $25-\mathrm{OH}-\mathrm{D}_{3}$ was intravenously administered to patients and positively correlated to increased $25-\mathrm{OH}-\mathrm{D}_{3}$ in urine (35). It is 
important to note that broilers grown to the grower phase for this study were fed common starter diets with $1 \alpha$ supplementation. When these broilers were sampled, broilers not fed $1 \alpha$ in the grower phase had a period of 18 days since they were given $1 \alpha$ supplementation. However, this may not impact our findings because in plasma, $1,25-(\mathrm{OH})_{2}-\mathrm{D}_{3}$ has a half-life of about $10-$ $20 \mathrm{~h}$ and $25-\mathrm{OH}-\mathrm{D}_{3}$ is about 15 days (14). Considering broilers not fed $1 \alpha$ had consistent plasma levels of $25-\mathrm{OH}-\mathrm{D}_{3}$, it is unlikely that the $1 \alpha$ they were fed from the starter diet was remaining in their bodies because it would have been converted to $1,25-(\mathrm{OH})_{2}-\mathrm{D}_{3}$ and excreted or used up before blood was collected.

Excess $25-\mathrm{OH}-\mathrm{D}_{3}$ in chickens is converted to $24,25-(\mathrm{OH})_{2}$ $\mathrm{D}_{3}$ by 24-hydroxylase in kidneys (36). $24,25-(\mathrm{OH})_{2}-\mathrm{D}_{3}$ is an inactive vitamin $\mathrm{D}$ form that is excreted, but this form is not the end-product of this pathway; $24,25-(\mathrm{OH})_{2}-\mathrm{D}_{3}$ goes through a series of conversions and ends up becoming calcitroic acid, a water-soluble molecule that is readily excreted (37). Although no difference in $24,25-(\mathrm{OH})_{2}-\mathrm{D}_{3}$ plasma concentration was observed, we speculate that the calcitroic acid concentration would be higher in $1 \alpha$-fed broilers because $1 \alpha$ cannot be converted to $24,25-(\mathrm{OH})_{2}-\mathrm{D}_{3}$. $1 \alpha$ would likely be converted to $1,25-(\mathrm{OH})_{2}-\mathrm{D}_{3}$ and then undergo multiple conversions to become calcitroic acid. We constructed a hypothetical model to compare how dietary $\mathrm{D}_{3}$ and $1 \alpha$ would be converted to calcitroic acid to be excreted (Figure 6). $1 \alpha$-fed broilers exhibiting a linear drop in $25-\mathrm{OH}-\mathrm{D}_{3}$ plasma concentration are a consequence of $1 \alpha$ being converted to $1,25-(\mathrm{OH})_{2}-\mathrm{D}_{3}$, which will reduce the amount of vitamin $\mathrm{D}_{3}$ being converted into 25-OH-D 3 due to a negative feedback loop (38). As Ca inclusion increased in $1 \alpha$-fed broilers, $25-\mathrm{OH}-\mathrm{D}_{3}$ concentration likely dropped because of $1 \alpha$ 's effects on $\mathrm{Ca}$ absorption. Purifying fecal content to measure vitamin D may provide insights to how much $\mathrm{D}_{3}$ was excreted relative to how much was fed.

For broilers sampled during the grower phase, broilers fed $0.54 \%$ Ca without $1 \alpha$ had about 0.18 relative expression increase of CALB compared to control ( $\mathrm{D}_{3}, 0.76 \% \mathrm{Ca}$ inclusion): this observation could signify that broilers were trying to bind dietary $\mathrm{Ca}$ for transport and absorption. Our results were similar to a different study by $\mathrm{Li}$ et al. (39) in which low Ca upregulated CALB expression. Although our results indicated MUC2 expression was statistically lower in $\mathrm{D}_{3}+1 \alpha$ broilers, relative expression was only decreased by about 0.5 compared to $\mathrm{D}_{3}$-fed broilers, which indicates no change in MUC2 expression. This finding is indicative that these broilers can be considered healthy, an inference supported by the FITC-D data, which denoted no difference in jejunal morphology.

VDR is responsible for signal transduction of Ca absorption genes when $1,25-(\mathrm{OH})_{2}-\mathrm{D}_{3}$ binds to it (40). For VDR expression in duodenum of 35-day old broilers, even though it was not

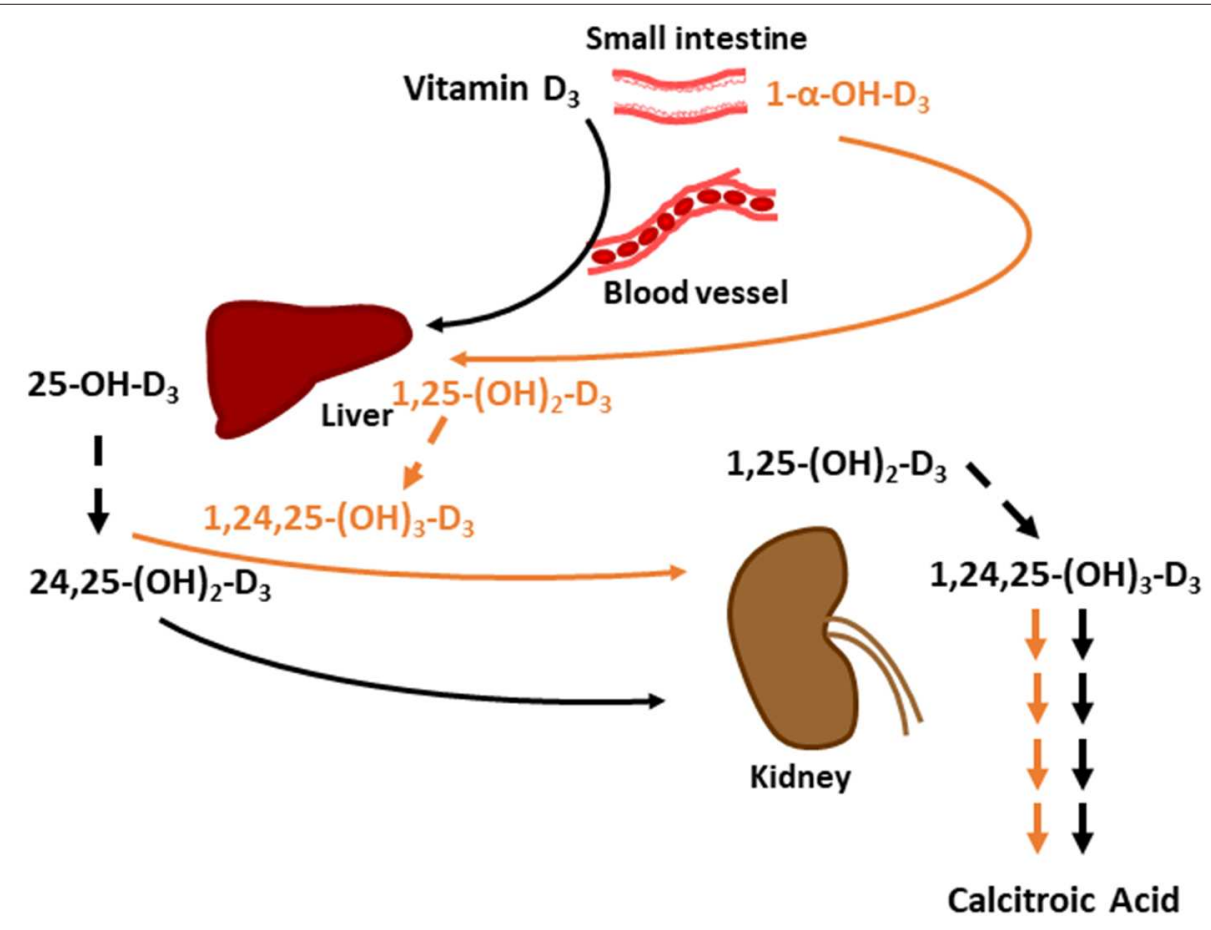

FIGURE 6 | Hypothetical model on comparing how dietary vitamin $D_{3}$ and 1 - $\alpha$-hydroxycholecalciferol (1 $\left.\alpha\right)$ are converted to water soluble calcitroic acid to be excreted. Black arrows denote vitamin $\mathrm{D}_{3}$ 's pathway to calcitroic acid and orange arrows denote $1 \alpha$ 's pathway. Dashed arrows signify 24 -hydroxylation step. Multiple arrows between 1,24,25- $(\mathrm{OH})_{3}-\mathrm{D}_{3}$ and calcitroic acid denote number of conversion steps. 25- $\mathrm{OH}-\mathrm{D}_{3}$ is $25-\mathrm{hydroxycholecalciferol}$; $24,25-(\mathrm{OH})_{3}-\mathrm{D}_{3}$ is 24,25-dihydroxycholecalciferol; $1,25-(\mathrm{OH})_{3}-\mathrm{D}_{3}$ is 1,25-dihydroxycholecalciferol; and1,24,25- $(\mathrm{OH})_{3}-\mathrm{D}_{3}$ is 1,24,25-trihydroxycholecalciferol. 


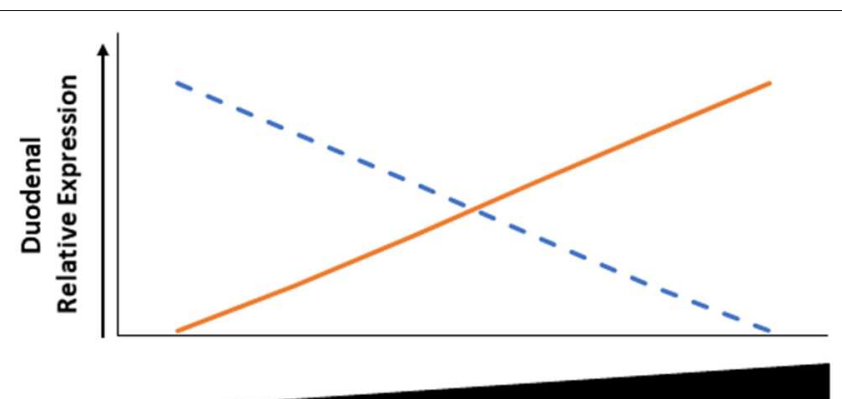

Calcium Availability

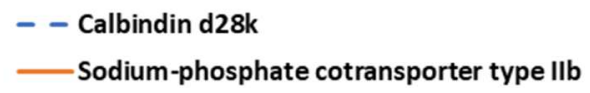

FIGURE 7 | Inverse relationship between duodenal calbindin d28k (CALB) and sodium-phosphate cotransporter type Illb (NPTIIb) in broiler chickens relative to calcium availability. As duodenal calcium concentration increases, then CALB expression decreases and NPTIlb expression increases to potentially maximize phosphate absorption because of the potential excessive calcium binding to phosphorus to form tricalcium phosphate and making phosphorus unavailable.

statistically different, a trending increase of VDR expression as $\mathrm{Ca}$ inclusion increased for broilers not fed $1 \alpha$ indicates dietary Ca's regulatory role with increasing VDR expression. VDR's role as a signal transducer for gene expression for proteins (TRPV6 and CALB) explains why vitamin $\mathrm{D}$ is important for dietary $\mathrm{Ca}$ absorption (41).

NPTIIb is an important cotransporter protein that requires sodium to move $\mathrm{P}$ in its phosphate form into cells from the intestinal lumen (42). Increasing Ca inclusion led to an increase in duodenal NPTIIb expression in 35-day $\mathrm{D}_{3}$ broilers, which was likely caused by $\mathrm{P}$ imbalance. Even though our study did not include citric acid, there was a study that noted how $1 \alpha$, phytase, and citric acid increased phytate $P$ utilization in broiler chicks (22). Excess dietary Ca compared to $P$ can cause $\mathrm{Ca}$ to bind to $P$ and form insoluble tricalcium phosphate (43). Our results were similar to Li et al. (39), in which increased dietary Ca levels led to increased NPTIIb expression in duodenum. Li et al. also noted how high dietary Ca could cause a decrease in available $P$, which triggers the increased expression of NPTIIb in the duodenum. A proposed model of the inverse relationship between CALB and NPTIIb expression relative to Ca inclusion levels is denoted for broilers not fed $1 \alpha$ (Figure 7). Further characterization of this relationship can elucidate the need to reevaluate nutrient interrelationships in animal production. There may be unintended impacts of using excessive nutrients as

\section{REFERENCES}

1. Bar A, Shinder D, Yosefi S, Vax E, Plavnik I. Metabolism and requirements for calcium and phosphorus in the fast-growing chicken as affected by age. Br J Nutr. (2003) 89:51-60. doi: 10.1079/BJN20 02757 a safety net for meeting nutrient requirements because potential deficiencies can be caused by nutrient antagonism, but these consequences can be detected by examining genes related to absorption such as NPTIIb and CALB.

Our work highlights that $1 \alpha$ supplementation with certain levels of $\mathrm{Ca}$ inclusion can impact blood $\mathrm{Ca}$ concentration and affect vitamin D metabolite concentration. Our findings exhibit that $1 \alpha$ can improve $\mathrm{Ca}$ utilization in young broilers with an implication for reducing dietary $\mathrm{Ca}$ in their diets. We suggest $1 \alpha$ should be supplemented in broiler diets for the starter phase and either removed for the grower phase or provided with reduced dietary $\mathrm{Ca}$ levels. 1 $\alpha$ 's potential to reduce dietary $\mathrm{Ca}$ without any negative impacts on growth performance signifies the importance of synthetic nutrients for improving animal production while reducing potential environmental impacts from excreted excess nutrients. Future research should explore how much $1 \alpha$ supplementation will cause vitamin $\mathrm{D}$ toxicity in growing animals to characterize an animal's regulatory limits of removing vitamin $\mathrm{D}$ with consideration of $1 \alpha$ 's bypassing of negative feedback regulation.

\section{DATA AVAILABILITY STATEMENT}

The raw data supporting the conclusions of this article will be made available by the authors, without undue reservation, to any qualified researcher.

\section{ETHICS STATEMENT}

The animal study was reviewed and approved by Institutional Animal Care and Use Committee, North Carolina State University.

\section{AUTHOR CONTRIBUTIONS}

MW, JF, and KL contributed to the conception and design of the study. MW and KL contributed to the acquisition, analysis, interpretation of data, wrote the first draft of the manuscript, and were responsible for the final editing of the manuscript. MW, TV, OT, and KL contributed to the manuscript. All authors read and approved the final manuscript.

\section{ACKNOWLEDGMENTS}

The authors would like to thank KL and Edens lab group members, Marilyn Mayer, and Catherine Lopez who gave feedback for the manuscript drafts. The authors would also like to thank Viviana San Martin Diaz for sharing the diet tables used in this study.

2. Waldenstedt L. Nutritional factors of importance for optimal leg health in broilers: a review. Anim Feed Sci Technol. (2006) 126:291-307. doi: 10.1016/j.anifeedsci.2005.08.008

3. Edwards H, Shirley R, Escoe W, Pesti G. Quantitative evaluation of 1-alphahydroxycholecalciferol as a cholecalciferol substitute for broilers. Poultry Sci. (2002) 81:664-9. doi: 10.1093/ps/81.5.664 
4. Sandström B. Micronutrient interactions: effects on absorption and bioavailability. Br J Nutr. (2001) 85:S181-5. doi: 10.1079/BJN2000312

5. Onderci M, Sahin N, Sahin K, Cikim G, Aydín A, Ozercan I, et al. Efficacy of supplementation of $\alpha$-amylase-producing bacterial culture on the performance, nutrient use, and gut morphology of broiler chickens fed a corn-based diet1. Poultry Sci. (2006) 85:505-10. doi: 10.1093/ps/85.3.505

6. Amerah AM, Gilbert C, Simmins PH, Ravindran V. Influence of feed processing on the efficacy of exogenous enzymes in broiler diets. World Poultry Sci J. (2011) 67:29-46. doi: 10.1017/S0043933911000031

7. Fallon MB, Boyer JL. Hepatic toxicity of Vitamin A and synthetic retinoids. J Gastroenterol Hepatol. (1990) 5:334-42. doi: 10.1111/j.1440-1746.1990.tb01635.x

8. Mortensen JT, Brinck P, Binderup L. Toxicity of vitamin D analogues in rats fed diets with standard or low calcium contents. Pharmacol Toxicol. (1993) 72:124-7. doi: 10.1111/j.1600-0773.1993.tb00302.x

9. Moritz J, Parsons A, Buchanan N, Baker N, Jaczynski J, Gekara O, et al. Synthetic methionine and feed restriction effects on performance and meat quality of organically reared broiler chickens. J Appl Poultry Res. (2005) 14:521-35. doi: 10.1093/japr/14.3.521

10. Haussler MR, Zerwekh JE, Hesse RH, Rizzardo E, Pechet MM. Biological activity of $1 \alpha$-hydroxycholecalciferol, a synthetic analog of the hormonal form of vitamin D3. Proc Natl Acad Sci USA. (1973) 70:2248-52. doi: $10.1073 /$ pnas.70.8.2248

11. Biehl RR, Baker DH, Deluca HF. 1 alpha-Hydroxylated cholecalciferol compounds act additively with microbial phytase to improve phosphorus, zinc and manganese utilization in chicks fed soy-based diets. J Nutr. (1995) 125:2407-16. doi: 10.1093/jn/125.9.2407

12. Edelstein S, Noff D, Freeman D, Sheves M, Mazur Y. Synthesis of $1 \alpha$-hydroxy [7-3H] cholecalciferol and its metabolism in the chick. Biochem J. (1978) 176:111-7. doi: 10.1042/bj1760111

13. Miller WL, Portale AA. Vitamin D 1 $\alpha$-Hydroxylase. Trends Endocrinol Metab. (2000) 11:315-9. doi: 10.1016/S1043-2760(00)00287-3

14. Jones G. Pharmacokinetics of vitamin D toxicity. Am J Clin Nutr. (2008) 88:582S-6S. doi: 10.1093/ajcn/88.2.582S

15. Hurwitz S, Plavnik I, Shapiro A, Wax E, Talpaz H, Bar A. Calcium metabolism and requirements of chickens are affected by growth. J Nutr. (1995) 125:2679-86.

16. Holick MF. Vitamin D and bone health. J Nutr. (1996) 126:1159S-64S. doi: 10.1093/jn/126.suppl_4.1159S

17. Wang J, Chen J-S, Zong J-Y, Zhao D, Li F, Zhuo R-X, et al. Calcium carbonate/carboxymethyl chitosan hybrid microspheres and nanospheres for drug delivery. J Phys Chem C. (2010) 114:18940-5. doi: 10.1021/jp105906p

18. Leach R Jr, Nesheim M. Nutritional, genetic and morphological studies of an abnormal cartilage formation in young chicks. J Nutr. (1965) 86:236-44. doi: $10.1093 /$ jn/86.3.236

19. Edwards HM Jr, Veltmann JR Jr. The role of calcium and phosphorus in the etiology of tibial dyschondroplasia in young chicks. J Nutr. (1983) 113:156875. doi: $10.1093 / \mathrm{jn} / 113.8 .1568$

20. Williams B, Solomon S, Waddington D, Thorp B, Farquharson C. Skeletal development in the meat-type chicken. Br Poultry Sci. (2000) 41:141-9. doi: $10.1080 / 713654918$

21. Han J, Wang J, Chen G, Zhang J, Zhang N, Qu H, et al. $1 \alpha-$ Hydroxycholecalciferol improves the growth performance and up-regulates the mRNA expression of vitamin D receptor in the small intestine and kidney of broiler chickens. Poultry Sci. (2018) 97:1263-70. doi: 10.3382/ps/pex423

22. Snow J, Baker D, Parsons C. Phytase, citric acid, and $1 \alpha-$ hydroxycholecalciferol improve phytate phosphorus utilization in chicks fed a corn-soybean meal diet. Poultry Sci. (2004) 83:1187-92. doi: $10.1093 / \mathrm{ps} / 83.7 .1187$

23. San Martin Diaz VE. Effects of 1-alpha-hydroxycholecaciferol and other Vitamin D analogs on live performance, bone development, meat yield and quality, and mineral digestibility on broilers. (MS Thesis). (2018).

24. Livak KJ, Schmittgen TD. Analysis of relative gene expression data using realtime quantitative PCR and the 2- $\Delta \Delta C T$ method. Methods. (2001) 25:402-8. doi: $10.1006 /$ meth.2001.1262

25. Siggaard-Andersen O, Fogh-Andersen N. Base excess or buffer base (strong ion difference) as measure of a non-respiratory acidbase disturbance. Acta Anaesthesiol Scandinavica. (1995) 39:123-8. doi: 10.1111/j.1399-6576.1995.tb04346.x
26. Mongin P. Recent advances in dietary anion-cation balance: applications in poultry. J Proc Nutr Soc. (1981) 40:285-94. doi: 10.1079/PNS19810045

27. Borges S, Da Silva AF, Maiorka A. Acid-base balance in broilers. World Poultry Sci J. (2007) 63:73-81. doi: 10.1017/S0043933907001286

28. Moore EW. Ionized calcium in normal serum, ultrafiltrates, and whole blood determined by ion-exchange electrodes. J Clin Investig. (1970) 49:318-34. doi: 10.1172/JCI106241

29. Shafey T, Mcdonald M, Pym R. Effects of dietary calcium, available phosphorus and vitamin $\mathrm{D}$ on growth rate, food utilisation, plasma and bone constituents and calcium and phosphorus retention of commercial broiler strains. Br Poultry Sci. (1990) 31:587-602. doi: 10.1080/00071669008417290

30. Broz J, Oldale P, Perrin-Voltz AH, Rychen G, Schulze J, Nunes CS. Effects of supplemental phytase on performance and phosphorus utilisation in broiler chickens fed a low phosphorus diet without addition of inorganic phosphates. Br Poultry Sci. (1994) 35:273-80. doi: 10.1080/00071669408417691

31. Sebastian S, Touchburn S, Chavez E, Lague P. Efficacy of supplemental microbial phytase at different dietary calcium levels on growth performance and mineral utilization of broiler chickens. Poultry Sci. (1996) 75:1516-23. doi: $10.3382 /$ ps.0751516

32. Galla JH. Metabolic alkalosis. J Am Soc Nephrol. (2000) 11:369-75.

33. Foy D, De Morais HA. Metabolic alkalosis: a quick reference. Vet Clin North Am. (2008) 38:435-8. doi: 10.1016/j.cvsm.2008.01.023

34. National Research Council Nutrient Requirements of Poultry. Washington, DC: National Academies Press. (1994).

35. Krawitt E, Grundman M, Mawer EB. Absorption, hydroxylation, and excretion of vitamin D3 in primary biliary cirrhosis. Lancet. (1977) 310:12469. doi: 10.1016/S0140-6736(77)92660-5

36. Shanmugasundaram R, Selvaraj R. Vitamin D-1 $\alpha$-hydroxylase and vitamin D24-hydroxylase mRNA studies in chickens. Poultry Sci. (2012) 91:1819-24. doi: 10.3382/ps.2011-02129

37. Reddy GS, Tserng KY. Calcitroic acid, end product of renal metabolism of 1 , 25-dihydroxyvitamin D3 through the C-24 oxidation pathway. Biochemistry. (1989) 28:1763-9. doi: 10.1021/bi00430a051

38. Tanaka Y, Lorenc RS, Deluca HF. The role of 1,25-dihydroxyvitamin D3 and parathyroid hormone in the regulation of chick renal 25hydroxyvitamin D3-24-hydroxylase. Archiv Biochem Biophys. (1975) 171:5216. doi: 10.1016/0003-9861(75)90061-2

39. Li J, Yuan J, Guo Y, Sun Q, Hu X. The influence of dietary calcium and phosphorus imbalance on intestinal NaPi-IIb and calbindin mRNA expression and tibia parameters of broilers. Asian-Australasian J Animal Sci. (2012) 25:552. doi: 10.5713/ajas.2011.11266

40. Van Cromphaut SJ, Dewerchin M, Hoenderop JG, Stockmans I, Van Herck E, Kato S, et al. Duodenal calcium absorption in vitamin D receptor-knockout mice: functional and molecular aspects. Proc Natl Acad Sci USA. (2001) 98:13324-9. doi: 10.1073/pnas.231474698

41. Cui M, Li Q, Johnson R, Fleet JC. Villin promoter-mediated transgenic expression of transient receptor potential cation channel, subfamily $\mathrm{V}$, member 6 (TRPV6) increases intestinal calcium absorption in wild-type and vitamin D receptor knockout mice. J Bone Mineral Res. (2012) 27:2097-107. doi: $10.1002 /$ jbmr.1662

42. Hilfiker H, Hattenhauer O, Traebert M, Forster I, Murer H, Biber J. Characterization of a murine type II sodium-phosphate cotransporter expressed in mammalian small intestine. Proc Natl Acad Sci USA. (1998) 95:14564-9. doi: 10.1073/pnas.95.24.14564

43. Heaney RP, Nordin BEC. Calcium effects on phosphorus absorption: implications for the prevention and co-therapy of osteoporosis. J Am College Nutr. (2002) 21:239-44. doi: 10.1080/07315724.2002.10719216

Conflict of Interest: The authors declare that this study received funding from Premex. The funder had the following involvement with the study: conception and design of the study.

Copyright (C) 2020 Warren, Vu, Toomer, Fernandez and Livingston. This is an openaccess article distributed under the terms of the Creative Commons Attribution License (CC BY). The use, distribution or reproduction in other forums is permitted, provided the original author(s) and the copyright owner(s) are credited and that the original publication in this journal is cited, in accordance with accepted academic practice. No use, distribution or reproduction is permitted which does not comply with these terms. 